\title{
EFEITO DA TERAPIA PERIODONTAL NÃO CIRÚRGICA NA REDUÇÃO DA SEVERIDADE DA ARTRITE REUMATOIDE
}

\section{EFFECT OF NON-SURGICAL PERIODONTAL THERAPY IN THE REDUCTION OF SEVERITY OF RHEUMATOID ARTHRITIS}

\author{
Renata Pereira de Sousa', Nayana Emanuelle Santos Figueiredoº \\ Mariana Vasconcelos Guimarães ${ }^{3}$ \\ 'Graduanda em Odontologia no Centro Universitário Doutor Leão Sampaio. Juazeiro do Norte, Ceará, Brasil. renatapsousa@hotmail.com \\ http://orcid.org/0000-0002-1490-5519 \\ ${ }^{2}$ Graduanda em Odontologia no Centro Universitário Doutor Leão Sampaio. Juazeiro do Norte, Ceará, Brasil. nayana.s.figueiredo@hotmail.com \\ http://orcid.org/0000-0001-6678-7748 \\ ${ }^{3}$ Professora no Centro Universitário Doutor Leão Sampaio. Juazeiro do Norte, Ceará, Brasil. marianav_guimaraes@yahoo.com.br \\ http://orcid.org/0000-0003-1086-8009
}

RESUMO | A remodelação óssea é um processo contínuo que depende da atuação equilibrada entre osteoclastos e osteoblastos, responsáveis pela reabsorção e formação óssea, respectivamente. Situações inflamatórias persistentes podem alterar este processo, como a artrite reumatoide (AR) e a periodontite crônica. A patogênese destas duas doenças está associada às alterações nos níveis de mediadores inflamatórios que leva a um desequilíbrio imunológico associado à perda óssea. Estudos têm sugerido que tais doenças são bidirecionais, onde a presença de uma influencia a evolução da outra. Esse trabalho revisa os resultados de estudos de pesquisas científicas sobre 0 efeito da terapia periodontal não cirúrgica (TP) na redução da gravidade da AR. Para tanto, foi realizada uma busca por artigos científicos publicados a partir do ano 2006, no banco de dados PubMed, utilizando-se as palavras chaves: artrite reumatoide, periodontite e tratamento periodontal, nos idiomas inglês/português. Foram selecionados nove estudos com metodologia de pesquisa semelhante e que compuseram amostras de pacientes acometidos pelas duas doenças. Parâmetros como índice de sangramento gengival, profundidade de sondagem, sangramento à sondagem ou nível clínico de inserção avaliaram a condição periodontal de cada paciente, enquanto que questionários averiguando a qualidade de vida dos pacientes e os testes laboratoriais relacionados à atividade inflamatória sistêmica foram considerados parâmetros associados à severidade da artrite reumatoide. Em oito estudos, a TP reduziu significamente severidade inflamatória da AR. Assim, os últimos dados científicos indicam o benefício da realização do TP ao reduzir a gravidade da AR, considerando, ainda a facilidade e baixo custo deste tipo de terapia.

\begin{abstract}
Bone remodeling is a continuous process that depends on the balanced action between osteoclasts and osteoblasts, responsible for reabsorption and bone formation, respectively. Persistent inflammatory conditions can alter this process, such as rheumatoid arthritis (RA) and periodontitis. The pathogenesis of these two diseases is associated with changes in the levels of inflammatory mediators leading to an immune imbalance associated with bone loss. Some studies have suggested that such diseases are bidirectional, where the presence of one influences the evolution of the other. This paper reviews the results of scientific research studies on the effect of non-surgical periodontal therapy (TP) on the reduction of RA severity. To do so, a search was made for scientific articles published from the year 2006, in the PubMed database, using the key words: rheumatoid arthritis, periodontitis and periodontal treatment, in the English/Portuguese languages. Nine studies with similar research methodology were selected and composed of samples from patients affected by both diseases. Parameters such as gingival bleeding index, depth of probing, bleeding at the probe or clinical level of insertion evaluated the periodontal condition of each patient, while questionnaires investigating patients' quality of life and laboratory tests related to systemic inflammatory activity were considered associated parameters To the severity of rheumatoid arthritis. In eight studies, PT significantly reduced inflammatory severity of RA. Thus, the latest scientific data indicate the benefit of TP by reducing the severity of RA, considering the ease and low cost of this type of therapy.
\end{abstract}

Key words: Rheumatoid arthritis. Periodontitis. Periodontal treatment.

Palavras-chave: Artrite reumatoide. Periodontite. Tratamento periodontal. 


\section{INTRODUÇÃO}

A periodontite crônica ( $P C)$, uma doença de alta prevalência e principal causa de perdas de dentes em adultos ${ }^{1,2}$, é uma resposta imunoinflamatória que acomete os tecidos de sustentação dos dentes, causando destruição de ligamento periodontal, de cemento radicular e de osso alveolar ${ }^{3}$. Apesar das investigações na patogênese dessa doença tenham se concentrado na infecção bacteriana durante muitas décadas, exaltando o biofilme dental como fator etiológico primário, nos últimos anos, uma maior influência tem sido atribuída à resposta do hospedeiro como fator fundamental na patogênese e na progressão da $\mathrm{PC}^{4}$. Nesse contexto, vale salientar que há um consenso bem estabelecido na literatura sobre a influência de fatores sistêmicos que esta doença pode sofrer ${ }^{5,6}$, podendo a PC ser exacerbada pelos mesmos e/ou interagir com eles.

Dentre os prováveis fatores sistêmicos envolvidos, a artrite reumatoide (AR) tem se destacado em decorrência de sua similaridade de mecanismos patogênicos à $P C^{7,8}$, o que pode justificar uma possível associação entre estas duas doenças. Trata-se de uma condição autoimune e, por vezes, inflamatória crônica, onde ocorre erosão óssea focal nas margens da articulação afetada?

Assim, o diagnóstico desta doença é estabelecido considerando-se os achados clínicos, como rigidez matinal e limitação de movimentos, além de exames complementares ${ }^{10}$.

O mecanismo patogênico da $P C$ e da AR está associado às alterações nos níveis de mediadores inflamatórios, que leva a um desequilíbrio imunológico relacionado à perda óssea ${ }^{11}$. De fato, alguns estudos na literatura científica têm demonstrado uma relação aproximada entre o metabolismo ósseo e o sistema imune, de tal forma que doenças inflamatórias crônicas, como estas duas, são caracterizadas por perda óssea ${ }^{11}$.

Logo, a literatura científica, com base em alguns estudos, tem sugerido que a $P C$ e a $A R$ podem ser consideradas como doenças que sofrem influências entre si, na medida em que a presença de uma condição inflamatória crônica influencia a progressão da outra, quando ambas se encontram no indivíduo $0^{6,7}$. Considerando a facilidade do tratamento periodontal não cirúrgico (TP) da PC, realizado por meio do debridamento mecânico e capaz de reduzir os sinais inflamatórios e a progressão da $\mathrm{PC}^{12}$, pareceu-nos ser importante averiguar $\circ$ que estudos científicos atuais na literatura têm a esclarecer sobre a influência da TP na gravidade dos sinais e sintomas clínicos da AR em pacientes acometidos também por PC.

\section{MÉTODO}

Neste trabalho, o efeito da TP na redução da gravidade da AR foi estudado através de revisão de literatura. Para isso, foi realizada uma busca por artigos científicos publicados a partir do ano 2006 no banco de dados PubMed, utilizando-se as palavras chaves: Artrite reumatoide, periodontite e tratamento periodontal, combinadas entre si, no idioma inglês.

Dentre 119 artigos científicos encontrados, foram selecionados apenas 08 , os quais foram publicados desde $\circ$ ano 2006 e avaliaram diretamente e prospectivamente $\circ$ efeito da TP na severidade da $A R$ em paciente com PC e AR. Foram excluídos desta pesquisa estudos de revisão de literatura, bem como aqueles que avaliaram ferramentas farmacológicas na evolução da $P C$ e/ou da $A R$, estudos retrospectivos ou que avaliaram apenas $\mathrm{e}$ exclusivamente as condições orais dos pacientes, sem averiguar, de alguma forma, a eficácia do TP na evolução da AR.

Todos os artigos selecionados para a presente revisão foram advindos de pesquisas científicas randomizadas, realizadas clinicamente em pacientes acometidos por AR e PC, em que frequentemente estes foram divididos em grupos de pacientes tratados e não tratados periodontalmente. Alguns desses estudos compuseram, ainda, um grupo controle onde os pacientes foram sistemicamente saudáveis e não apresentaram a doença. Os parâmetros utilizados para analisar a atividade destas duas doenças 
foram semelhantes entre tais pesquisas científicas e os mesmos foram averiguados em diferentes períodos destes estudo, de forma prospectiva, significando que tais pacientes apresentaram um certo período de acompanhamento para se obter os resultados.

\section{REVISÃO DE LITERATURA}

\section{PERDA ÓSSEA INFLAMATÓRIA NA ARTRITE REUMATOIDE E NA PERIODONTITE CRÔNICA}

A AR é uma doença inflamatória crônica que acomete essencialmente as articulações, embora outros órgãos também possam estar comprometidos ${ }^{13}$. Por se tratar de uma condição auto-imune, sabe-se que - sistema imunitário do organismo é direcionado contra aos seus próprios tecidos ${ }^{14}$. $O$ aparecimento desta doença decorre de vários fatores, os quais incluem a predisposição genética, a exposição a fatores ambientais e, possivelmente, às infecções ${ }^{10}$.

Dentre estas etiologias, a mais provável é a tendência genética $e$, de fato, acredita-se que alguns genes possam interagir com os outros fatores causais da doença ${ }^{10}$. Apesar desse conhecimento, vale salientar que contrariamente alguns pacientes acometidos com a doença não apresentam estes genes, bem como a presença destes genes também não significa que o portador irá sempre demonstrar sinais clínicos ${ }^{10}$. Além dos fatores genéticos, inúmeros vírus e bactérias foram investigados como também sendo possíveis causadores da doença ${ }^{15,16}$. Neste contexto, vale ainda salientar que doenças inflamatórias crônicas, tal como a PC, podem interagir sinergicamente com a $A R^{17}$.

O processo inflamatório da AR caracteriza-se pela hiper-regulação e liberação de citocinas pró-inflamatórias, como $\circ$ fator de necrose tumoral- $\alpha$ (TNF- $\alpha$ ) e interleucinas (IL)- 1 e IL- $6^{18}$, culminando na destruição da cartilagem articular e do osso subcondral ${ }^{16}$. A presença sustentada destes mediadores solúveis e de outras moléculas efetoras da inflamação acarreta na degradação do colágeno e das moléculas proteoglicanas, seja de forma direta ou indireta. Além disso, há também a produção de prostaglandina (PG)- E2, de enzimas produzidas pelos neutrófilos, como a elastase e a $\beta$-glucuronidase, bem como a secreção de metaloproteinases da matriz (MMPs) ${ }^{19,20}$ pelos macrófagos e sinoviócitos, que contribuem também, de forma significativa, para a patogênese da $A R^{19}$.

Já a ativação osteoclástica tem como uma das origens alterações no eixo RANK/RANKL/OPG. Quanto a este assunto, sabe-se que a molécula de RANKL (ligante do receptor ativador do fator nuclear Kappa- $\beta$ ), a qual pode estar na forma solúvel ou expressa em inúmeros tipos celulares, sobretudo em osteoblastos, fibroblastos e linfócitos $\mathrm{T}^{11,21}$, ao ligar-se ao seu receptor RANK, ativa a via do factor nuclear Kappa- $\beta$, com consequente transcrição e expressão genética dos osteoclastos, constituindo em fatores chaves na regulação da formação e ativação destas células responsáveis pela reabsorção de tecido ósseo ${ }^{21}$. Por outro lado, a OPG (osteoprotegerina), uma molécula solúvel presente no plasma, é considerada um inibidor natural do RANKL, uma vez que age como antagonista desta ligação ao prevenir a ligação de RANKL ao RANK localizado na superfície dos percursores osteoclásticos, regulando o metabolismo ósseo ${ }^{21}$.

Estudos sugerem que células inflamatórias como os linfócitos $T$, presentes no líquido sinovial em articulações acometidas pela $A R$, constituem a principal fonte de RANKL nesta doença ${ }^{22,23,24}$.

Consequentemente, tal processo inflamatório é responsável por desregular o eixo RANK/RANKL/ OPG no sentido de aumentar a proporção RANKL/ OPG, fator este responsável pela observação do aumento da reabsorção óssea na $A R^{22}$. Além disso, - processo inflamatório crônico desta patologia estaria, também, associado à diminuição de OPG devido a apoptose induzida de osteoblastos ${ }^{22}$.

Dentre as doenças inflamatórias caracterizadas por perda óssea, além $A R$, onde ocorre erosão óssea focal nas margens da articulação afetada ${ }^{25}$, destaca-se a PC, que é acompanhada por destruição óssea alveolar inflamatória ${ }^{26}$. É bem verdade que algumas doenças inflamatórias tem a degradação óssea como uma de suas consequências ${ }^{27}$, de forma que $\circ$ grau de resposta inflamatória parece estar ligado à extensão da perda óssea, o que indica 
que a inibição de citocinas pró-inflamatorias podem reduzir o dano ósseo ${ }^{25,27,28,29,30}$. Assim, se estabelece uma relação entre os sistemas imunológico e ósseo mais estreita do que se parece.

Apesar da similaridade dos mecanismos patogênicos entre $A R$ e $P C$, a $P C$ consiste em processos inflamatórios não associados a desordens autoimunes, mas sim de origem infecciosa e inflamatória crônica, com acometimento dos tecidos gengivais e/ou os tecidos de suporte dos dentes. Assim, tais doenças ocorrem como consequência das reações inflamatórias e imunológicas dos tecidos periodontais induzidas pelo desafio microbiano decorrente da presença crônica do biofilme dental (placa bacteriana), culminando em perda de tecido ósseo alveolar e conjuntivo ${ }^{31,32}$, o que ocorre de forma progressiva lenta, assintomática e de caráter irreversível ${ }^{33}$.

Nesse contexto, sabendo que a evolução e a extensão do dano periodontal estão relacionadas à resposta imunoinflamatória exacerbada do hospedeiro, vale salientar que o osso é um tecido extremamente dinâmico e ativo, que sofre remodelação constante em resposta a influências mecânicas, nutricionais e hormonais $^{11}$, e tal processo envolve um equilíbrio entre os processos acoplados de reabsorção do osso por osteoclastos e de formação do osso por osteoblastos ${ }^{11}$. Sabe-se que tais células são produzidas por inúmeros fatores complexamente regulados e que um desequilíbrio em sua formação e/ou atividades pode acarretar alteração no saldo líquido final do processo de remodelação óssea ${ }^{34}$. Assim, em situações inflamatórias, por exemplo, onde - aumento da concentração de mediadores próinflamatórios culmina em ativação de osteoclastos e inibição de osteoblastos, observa-se alteração do processo de remodelação óssea, resultando em perda óssea ${ }^{34}$.

Desta forma, a participação de TNF- $\alpha$, IL-6, IL-1 $\beta$, IL-17 e PGE2 promove a indução da reabsorção óssea indiretamente através da alteração do controle do sistema RANK/RANKL/OPG, por aumentar a razão RANKL/OPG ${ }^{11}$, e diretamente, uma vez que mediadores inflamatórios possuem efeitos antiapoptóticos diretos sobre osteoclastos, prolongando sua vida útil, e estimulam sua formação através da atuação em seus precursores estromais ${ }^{11}$.
De fato, como demonstrado anteriormente, células estaminais hematopoiéticas, precursoras de osteoclastos, possuem receptores passíveis de ligação por tais citocinas ${ }^{11}$.

Considerando que PC e AR são caracterizadas pela destruição crônica de tecido ósseo associada a uma desregulação imunológica pelo aumento da liberação de citocinas pró-inflamatórias como IL-1, TNF- $\alpha$ e IL-6, além de PGE2, local e sistemicamente, determinados estudos científicos buscam averiguar se a PC apresenta alguma influência quanto à evolução e ao mecanismo patogênico da AR. Embora haja, na literatura, muitos autores que avaliem essa relação diretamente, a presente revisão a estuda através de resultados de pesquisas científicas que analisem o efeito da TP na redução da gravidade da AR. Isso porque tem-se conhecimento de que o biofilme bacteriano é o fator etiológico relacionado ao início deste processo inflamatório crônico e destrutivo da PC28, e, assim, estratégias simples para evitar o seu acúmulo por meio de uma boa higiene oral e de raspagem e alisamento radicular, que constituem a $T P$, ao serem empregadas, propiciam redução da carga microbiana e do processo inflamatório na $\mathrm{PC}^{12}$.

\section{EFEITO DA TERAPIA PERIODONTAL NÃO CIRÚRGICA NA REDUÇÃO DA SEVERIDADE DA ARTRITE REUMATOIDE}

Partindo do pressuposto que a AR é uma doença caracterizada por acometimento de articulações e que pode estar diretamente associada à qualidade de vida dos pacientes acometidos, decorrente da dor relacionada à atividade de seu processo inflamatório, verifica-se, na literatura, estudos de pesquisa científica utilizando questionários que avaliam o quesito qualidade de vida, bem como testes laboratoriais ou, até mesmo, correlações de índices de atividade da $A R$, visando categorizar o paciente em diferentes estratos de atividades da referida doença ${ }^{35}$. Os estudos selecionados para a presente revisão e que avaliaram o efeito da TP na redução da gravidade da AR utilizaram, em sua totalidade, metodologias semelhantes, dividindo os pacientes diagnosticados com estas duas doenças em grupos não tratados (controle) e tratados (teste). Assim, no estudo de AL-KATMA et al., $2007^{36}$, a 
atividade da $A R$ foi avaliada usando especialmente o disease activity score 28 (DAS28), além do teste laboratorial que analisa a taxa de sedimentação de eritrócitos (ESR). O DAS28 é um exame que avalia a gravidade desta doença com base na presença ou não de dor em 28 articulações do corpo humano ${ }^{37,38}$, enquanto que o teste laboratorial ESR é realizado por ser útil para avaliar a presença de inflamação sistêmica, de modo que quanto mais elevados os valores para este teste, maior o grau de inflamação. Neste estudo, observou-se melhoria do DAS28 no decorrer de 8 semanas após o TP em comparação a indivíduos não tratados. Além desta diferença significativa, o teste ESR também foi estatisticamente melhor para o grupo teste em comparação àquele controle.

Este resultado promissor também pôde ser encontrado no estudo de PINHO et al., 200939, que utilizou parâmetros clínicos, como profundidade de sondagem, placa microbiana e sangramento à sondagem para avaliação da atividade da PC, e os parâmetros DAS28 e Medical Outcomes Study 36 - Item Short - Form Health Survey (SF36) para avaliação da atividade da AR. A saber, o SF36 é um questionário com 36 itens que avalia a qualidade de vida de acordo com capacidade funcional, aspectos físicos, dor, estado geral da saúde, vitalidade, aspectos sociais, aspectos emocionais e saúde mental ${ }^{37}$. Além disso, também foi realizado, por tais autores, para cada paciente, $O$ teste laboratorial para proteína $C$ reativa $(P C R)^{40}$, que de forma semelhante ao ESR avalia a presença de inflamação sistêmica. Comparando os dois grupos de estudo, observou-se diferenças significantes para DAS28 após 3 meses do TP no grupo teste em comparação ao grupo controle, embora não foram verificadas diferenças significantes para PCR e ESR.

No mesmo ano, seguindo a mesma linha de raciocínio, ORTIZ et al.,2009 ${ }^{41}$, com o intuito de examinar o efeito do TP sobre os sinais e sintomas da artrite reumatoide, selecionaram 40 participantes com diagnóstico de AR moderada/grave e PC severa. Os parâmetros profundidade de sondagem, perda de inserção clínica, sangramento à sondagem e índices de placa foram úteis para avaliação periodontal, enquanto que os exames DAS-28 e ESR serviram para avaliação da AR. Observou-se que o grupo de indivíduos que receberam TP apresentou diminuição significativa na média DAS28 e na sorologia para TNF- $\alpha$, em comparação àqueles pacientes que não foram tratados periodontalmente, embora a redução na média de ESR foi insignificante.

Três anos mais tarde, esse assunto foi novamente estudado por ERCIYAS et al., $2012^{42}$, que utilizaram metodologia semelhante a estudos anteriores. Assim, a gravidade da AR foi avaliada por meio do DAS28, $E R S, P C R$, além de níveis de TNF- $\alpha$ sérico, enquanto que parâmetros periodontais convencionais já citados anteriormente para outros estudos foram também avaliados. Tais parâmetros, tanto da AR quanto da $P C$, apresentaram redução semelhante $e$ significante após 3 meses do tratamento periodontal convencional.

Até então, o DAS28 já estava presente como o principal índice clínico de atividade da AR dentre as pesquisas científicas relacionadas a tal assunto. De fato, foi o único parâmetro clínico relacionado a AR utilizado por BASAK et al., $2013^{43}$, os quais observaram melhoria do DAS28 em pacientes com AR tratados periodontalmente de forma convencional, sugerindo que há um sinergismo da TP com o quadro clínico da AR.

No mesmo ano, OKADA et al., $2013^{44}$, realizaram a avaliação dos efeitos da terapia periodontal não-cirúrgica na evolução da artrite reumatoide com um aprimoramento metodológico. Esse autores utilizaram parâmetros periodontais e reumatológicos, bem como níveis séricos de citocinas e marcadores inflamatórios como citrulina e imunoglobulina (IG) G para Porphyromonas gingivalis no início do estudo e 8 semanas após a TP. A saber, a citrulina não é um aminoácido-padrão, e sua presença em proteínas deve-se a uma modificação pós-traducional de proteínas contendo arginina, a qual será convertida à citrulina nas proteínas. Assim, pacientes com AR possuem uma variedade de auto-anticorpos no soro e no líquido sinovial. Entre esses autoanticorpos, destacam-se aqueles direcionados a proteínas citrulinadas, que são específicos para $A R$ e são úteis para auxiliar no diagnóstico da doença $e$ determinação da sua atividade ${ }^{45}$. Nesse estudo, observou-se que os níveis séricos de IGG para Porphyromonas gingivalis foram significativamente correlacionados positivamente com as de anticorpos citrulinados. Estas observações podem refletir um 
papel de Porphyromonas gingivalis na citrulinação de proteínas, a qual, por sua vez, está relacionada com a patogênese da AR. Embora tais resultados, neste estudo não se obsevaram diferenças significantes no PCR entre os grupos controle e tratado.

Apesar de os resultados destes estudos estivessem, em maioria, corroborando para a associação entre PC e AR, KURGAN et al., $2015^{46}$ obtiveram dados divergentes. Tais autores avaliaram os efeitos da TP sobre os níveis de metaloproteinase de matriz (MMP)-8, bem como de mediadores inflamatórios IL-6 e PGE2 no fluido gengival crevicular de em pacientes com AR e com PC. A atividade da AR foi avaliada através de $P C R, E S R$ e fator reumatoide. Tais autores observaram que as alterações significantes nos biomarcadores no fluido crevicular gengival não acompanharam melhorias importantes naqueles relacionados à atividade da $A R$, diferente do que foi observado na maioria dos achados já citados anteriormente em nosso estudo.

Embora tal resultado contrário, mais recentemente, KHARE et al., 201647, com o mesmo intuito, realizaram um estudo cujos parâmetros periodontais foram avaliados por meio do registro periodontal simplificado (PSR), índice de sangramento gengival, sangramento à sondagem, profundidade de sondagem, nível clínico de inserção. Enquanto que a $A R$ foi avaliada por parâmetros, como número de articulações inchadas, doloridas (DAS28), ESR, PCR e escala visual analógica de dor, os quais apresentaram-se significante reduzidos após tratamento periodontal.

Dentre os estudos selecionados para a presente revisão $(n=8)$, ○ principal meio de avaliação da gravidade da AR foi $\circ$ disease activity score 28 (DAS28) ( $n=7)$. Isso porque deve se considerar que a atividade da $A R$ é melhor avaliada por uma pontuação composta, em vez de análise individual de marcadores inflamatórios e clínicos, sendo este fato explicado pela natureza complexa da RA. Interessantemente, este parâmetro apresentou diferenças significativas entre grupos controle e teste, indicando que o controle da atividade inflamatória na PC por TP atenua a resposta inflamatória sistêmica que, por sua vez, contribui para melhorar a atividade de RA.

\section{CONSIDERAÇÕES FINAIS}

A TP em pacientes com artrite reumatoide $e$ periodontite, de acordo com a maioria dos relatos científicos sobre tal assunto e publicados a partir de 2006, pode ser promissora no quesito em contribuir para redução da gravidade da doença sistêmica $A R$ e, consequentemente, para melhoria da qualidade de vida destes pacientes.

\section{CONTRIBUIÇÕES DOS AUTORES}

Sousa RP participou da busca, leitura e seleção dos artigos científicos, confecção redação do artigo científico. Figueiredo NE participou da busca, leitura e seleção dos artigos científicos, confecção redação do artigo científico. Guimarães MV participou da análise dos artigos selecionados, orientação da redação do artigo científico.

\section{CONFLITOS DE INTERESSES}

Nenhum conflito financeiro, legal ou político envolvendo terceiros (governo, empresas e fundações privadas, etc.) foi declarado para nenhum aspecto do trabalho submetido (incluindo mas não limitandose a subvenções e financiamentos, conselho consultivo, desenho de estudo, preparação de manuscrito, análise estatística, etc).

\section{REFERÊNCIAS}

1. Eke PI, Zhang X, Lu H, Wei L, Thornton-Evans G, Greenlund $\mathrm{KJ}$ et al. Predicting Periodontitis at State and Local Levels in the United States. J Dent Res. 2016;95(5):515-522. doi: $10.1177 / 0022034516629112$

2. Holde GE, Oscarson N, Trovik TA, Tillberg A, Jönsson B. Periodontitis Prevalence and Severity in Adults: A Cross Sectional Study in Norwegian Circumpolar Communities. J Periodontol. 2017;3:1 -17. doi: 10.1902/iop.2017.170164

3. Armitage GC. Clinical evaluation of periodontal diseases. Periodontol 2000.1995;7:39-53.

4. Silva N, Abusleme L, Bravo D, Dutzan N, Garcia-Sesnich J, Vernal $\mathrm{R}$ et al. Host response mechanisms in periodontal diseases. J Appl Oral Sci. 2015;23(3):329-55. doi: $10.1590 / 1678-775720140259$

5. Nazir MA. Prevalence of periodontal disease, its association with systemic diseases and prevention. Int J Health Sci (Qassim). 2017;1 1 (2):72-80.

6. Monsarrat P, Vergnes JN, Blaizot A, Constantin A, Grado 
GF, Ramambazafy $\mathrm{H}$ et al. Oral health status in outpatients with rheumatoid arthritis: the OSARA study. Oral Health Dent Manag. 2014;13(1):113-9.

7. Venkataraman A, Almas K. Rheumatoid Arthritis and Periodontal Disease. An Update. N Y State Dent J. 2015;81(5):30-6.

8. Domínguez-Pérez RA, Loyola-Rodriguez JP, AbudMendoza C, Alpuche-Solis AG, Ayala-Herrera JL, MartínezMartínez RE. Association of cytokines polymorphisms with chronic peridontitis and rheumatoid arthritis in a Mexican population. Acta Odontol Scand. 2017;75(4):243-248. doi: 10.1080/00016357.2017.1280846

9. Goeldner I, Skare TL, Reason ITM, Utiyama SRR. Rheumatoid arthritis: a current view. J Bras Patol Med Lab. 2011 ;47(5):495-503. doi: 10.1590/S1676$\underline{24442011000500002}$

10. Mota LMH, Cruz BA, Brenol CV, Pereira IA, Fronza LSR, Bertolo $M B$ et al. Consenso da Sociedade Brasileira de Reumatologia 2011 para o diagnóstico e avaliação inicial da artrite reumatoide. Rev Bras Reumatol. 2011 ;51(3):207-19. doi: $10.1590 /$ S0482-50042011000300002

11. Pacifici R. The immune system and bone. Arch Biochem Biophys. 2010; 503(1):41-53. doi: 10.1016/i. abb.2010.05.027

12. Drisko $\mathrm{CH}$. Nonsurgical periodontal therapy. Periodontol 2000. $2001 ; 25: 77-88$.

13. Conigliaro $P$, Chimenti MS, Triggianese $P$, Sunzini $F$, Novelli $L$, Perricone $C$ et al. Autoantibodies in inflammatory arthritis. Autoimmun Rev. 2016;15(7):673-83. doi: 10.1016/i. autrev.2016.03.003

14. Souza AWS, Mesquita Júnior D, Araújo JAP, Castelan TTT, Cruvinel WM, Andrade LEC et al. Sistema Imunitário - Parte III O delicado equilíbrio do sistema imunológico entre os pólos de tolerância e autoimunidade. Rev Bras Reumatol. 2010;50(6):665-79. doi: 10.1590/S0482$\underline{50042010000600007}$

15. Guo ZY, Zhang JX, Wu M, Mei YF, Lin XJ, Bu C et al. Meta-analysis of the association between PADI4 -92C/G polymorphism and rheumatoid arthritis in the Chinese population. Braz J Med Biol Res. 2017;50(10):e61 15. doi: $\underline{10.1590 / 1414-431 \times 20176115}$

16. Mejía CR, López-Vélez R. Alfavirus tropicales artritogénicos. Reumatol Clin. 2017. doi: 10.1016/i. reuma.2017.01.006

17. Braga FSFF, Miranda LA, Miceli VC, Áreas A, Figueiredo CMS, Fischer RG et al. Artrite crônica e periodontite. Rev. Bras. Reumatol. 2007;47(4):276-8. doi: 10.1590/S0482$\underline{50042007000400006}$
18. Tanaka Y. Current concepts in the management of rheumatoid arthritis. Korean J Intern Med. 2016;31(2):210-8. doi: $\underline{10.3904 / \mathrm{kjim} .2015 .137}$

19. Angelotti F, Parma A, Cafaro G, Capecchi R, Alunno $A$, Puxeddu I. One year in review 2017: pathogenesis of rheumatoid arthritis. Clin Exp Rheumatol. 2017;35(3):368378.

20. Itoh Y. Metalloproteinases in Rheumatoid Arthritis: Potential The Therapeutic Targets to Improve Current Therapies. 2017;1 48:327-338. doi: 10.1016/ bs.pmbts.2017.03.002

21. Manara $M$, Sinigaglia L. Bone and TNF in Rheumatoid Arthritis: Clinical Implications. RMD Open. 2015; 1 (Suppl 1):e000065. doi: 10.1136/rmdopen-2015-000065

22. Amarasekara DS, Yu J, Rho J. Bone Loss Triggered by the Cytokine Network in Inflammatory Autoimmune Diseases. J Immunol Res. 2015;2015:8321 27. doi: $\underline{10.1155 / 2015 / 832127}$

23. Romas E, Gillespie MT, Martin TJ. Involvement of receptor activator of NFkappaB ligand and tumor necrosis factoralpha in bone destruction in rheumatoid arthritis. Bone. 2002;30(2):340-6.

24. Savola P, Kelkka T, Rajala HL, Kuuliala A, Kuuliala K, Eldfors $S$ et al. Somatic mutations in clonally expanded cytotoxic T lymphocytes in patients with newly diagnosed rheumatoid arthritis. Nat Commun. 2017;8:15869. doi: $10.1038 /$ ncomms 15869

25. Nanjundaiah SM, Astry B, Moudgil KD. Mediators of Inflammation-Induced bone damage in arthritis and their control by herbal products. Evid Based Complement Alternat Med. 2013;2013:518094. doi: 10.1155/2013/518094

26. Cochran DL. Inflammation and Bone Loss in Periodontal Disease. J periodontal. 2008;79(8 Suppl):1569-76. doi: 10.1902/iop.2008.080233

27. Hardy R, Cooper MS. Bone loss in inflammatory disorders Journal of endocrinology. 2009;201(3):309-20. doi: $\underline{10.1677 / J O E-08-0568}$

28. Taubman $M$, Valverde $P$, Han X, Kawai T. Immune response: The key to bone resorption in periodontal disease. J periodontal. 2005;76(11 Suppl):2033-41. doi: 10.1902/ ¡op.2005.76.11-S.2033

29. Mundy MD, Gregory R. Osteoporosis and inflammation. nutrition reviews. 2007;65(3):147-151. doi: $10.1111 / \mathrm{i} .1753$ 4887.2007.tb00353.x

30. Yucel L, Bage T. Inflammatory mediators in the pathogenesis of periodontitis. Expert Rev Mol Med. 2013;15:e7. doi: 10.1017/erm.2013.8 
31. Hienz SA, Paliwal S, Ivanovski S. Mechanisms of Bone Resorption in Periodontitis.J Immunol Res. 2015:615486. doi: $10.1155 / 2015 / 615486$

32. Bartold PM, Van Dyke TE. Periodontitis: a host-mediated disruption of microbial homeostasis. Unlearning learned concepts. Periodontology 2000. 2013;62(1):203-17. doi: $10.1111 / \mathrm{i} .1600-0757.2012 .00450 . x$

33. Preshaw PM, Alba AL, Jepsen S, Antonis K, Makrilakis $\mathrm{K}$ et al. Periodontitis and diabetes: a two-way relationship. Diabetologia. 201 2;55(1):21-31. doi: 10.1007/s00125$011-2342-y$

34. Cekici A, Kantarci A, Thomas E, Dyke V. Inflammatory and immune pathways in the pathogenesis of periodontal disease. Periodontol 2000. 2014;64(1):57-80. doi: 10.1111/ prd.12002

35. Medeiros MMC, Oliveira BMGB, Cequeira JVM, Quixadá BTS, Oliveira IMX. Correlacão dos índices de atividade da artrite reumatoide (Disease Activity Score 28 medidos com VHS, PCR, Simplified Disease Activity Index e Clinical Disease Activity Index) e concordância dos estados de atividade da doenca com vários pontos de corte numa população do nordeste brasileiro. Rev bras reumatol. 2015;5(6):477-484.

36. Al-Katma MK, Bissada NF, Bordeaux JM, Sue J, Askari AD. Control of periodontal infection reduces the severity of active rheumatoid arthritis. J Clin Rheumatol. 2007;13(3):134-7. doi: 10.1097/RHU.0b013e3180690616

37. Diniz LR, Balsamo S, Souza TY, Muniz LF, Martins WR, Mota LMHD. Measuring fatigue with multiple instruments in a Brazilian cohort of early rheumatoid arthritis patients. Rev Bras Reumatol Engl Ed. 2017;S2255-5021(17)30035-4. doi: 10.1016/i.rbre.2017.05.004

38. Schoels M, Alasti F, Smolen JS, Aletaha D. Evaluation of newly proposed remission cut-points for disease activity score in 28 joints (DAS28) in rheumatoid arthritis patients upon IL-6 pathway inhibition. Arthritis Res Ther. 2017;19(1):155. doi: 10.1186/s13075-017-1346-5

39. Pinho MNP, Oliveira RDR, Novaes Jr AB, Voltarelli JC. Relationship between periodontitis and rheumatoid arthritis and the effect of non-surgical periodontal treatment. Braz Dent J. 2009;20(5):355-64.

40. Pincus T, Sokka T. Laboratory tests to assess patients with rheumatoid arthritis: advantages and limitations. Rheum Dis Clin North Am. 2009;35(4):731-4, vi-vii. doi: 10.1016/i. rdc.2009.10.007

41. Ortiz P, Bissada NF, Palomo L, Han YW, Al-Zahrani MS, Panneerselvam $A$ et al. Periodontal therapy reduces the severity of active rheumatoid arthritis in patients treated with or without tumor necrosis factor inhibitors. J Periodontol. 2009;80(4):535-40. doi:10.1902/iop.2009.080447
42. Erciyas K, Sezer U, Ustün K, Pehlivan Y, Kisacik B, Senyurt $\mathrm{SZ}$ et al. Effects of periodontal therapy on disease activity and systemic inflammation in rheumatoid arthritis patients. Oral Dis. 2013;19(4):394-400. doi: 10.1111 /odi.12017

43. Basak B, Buduneli N, Nalbantsoy A, Lappin D, Evrenosoglu $E$, Kinane D. Periodontal therapy in chronic periodontitis lowers gingival crevicular fluid interleukin- 1 beta and DAS28 in rheumatoid arthritis patients. Rheumatol Int. 33(10):260716. doi: $10.1007 / \mathrm{s} 00296-013-2781-5$

44. Okada M, Kobayashi T, Ito S, Yokoyama T, Abe A, Murasawa A, Yoshie H. Periodontal treatment decreases levels of antibodies to Porphyromonas gingivalis and citrullinein patients with rheumatoid arthritis and periodontitis. J Periodontol. 2013;84(12):e74-84. doi: 10.1902/ jop.2013.130079

45. Alarcon RT, Andrade LEC. Anticorpos Antiproteínas Citrulinadas e a Artrite Reumatóide. Rev bras reumatol. São Paulo. 2007;47(03):1 80-187. doi: 10.1590/S0482$\underline{50042007000300007}$

46. Kurgan Ș, Fento Ö, Önder C, Serdar M, Eser F, Tatakis $\mathrm{DN}$ et al. The effects of periodontal therapy on gingival crevicular fluid matrix metalloproteinase-8, interleukin-6 and prostaglandin E2 levels in patients with rheumatoid arthritis. J Periodontal Res. 2016;51(5):586-95. doi: 10.1111/ jre. 12337

47. Khare N, Vanza B, Sagar D, Saurav K, Chauhan R, Mishra S. Nonsurgical Periodontal Therapy decreases the Severity of Rheumatoid Arthritis: A Case-control Study. J Contemp Dent Pract. $2016 ; 17(6): 484-8$. 